\title{
Arduino ve LabVIEW Kullanarak EMG Verilerinden Eşik Seviye Belirleme ile Motor Kontrol Düzeneği Tasarımı
}

\author{
Savaş ŞAHİN*1, Mutlu BAYRAKTAR², Ali Emre KAVUR², Kübra EVREN ȘAHİN
}

1İzmir Kâtip Çelebi Üniversitesi, Mühendislik Fakültesi, Elektrik-Elektronik Mühendisliği Bölümü, 35620, İZMİR

2 İzmir Kâtip Çelebi Üniversitesi, Fen Bilimleri Enstitüsü, Biyomedikal Teknolojileri Bölümü, 35620, İZMİR

${ }^{3}$ Dr. Behçet Uz Çocuk Hastanesi, 35210, İZMİR

(Alınış / Received: 02.05.2017, Kabul / Accepted: 25.08.2017, Online Yayınlanma / Published Online: 27.10.2017)

\section{Anahtar Kelimeler}

EMG,

LabVIEW,

Arduino,

Rehabilitasyon
Özet: Biyomedikal sinyaller içinde Elektromiyografinin (EMG) incelenmesi popüler bir araştırma alanıdır ve rehabilitasyon tasarım sistemleri için oldukça önemidir. Bu çalışmada kullanılan yöntemler sırasıyla; i) insan üzerinden iskelet kasının sinir yolu ile uyarılarak gerçek zamanlı el parmaklarına ait EMG verilerinin alınması, ii) elde edilen EMG işaretlerinden el açma ve kapama hareketlerinin eşik seviyelerinin histogram yöntemiyle belirlenmesi iii) eşik gerilimlerini kullanarak Arduino kartı üzerinden gerçek zamanlı DC motor kontrolü yapılmıştır. Bu çalışma da belirlenen eșik gerilimleri kullanılarak Arduino tabanlı bir devre ile rehabilitasyon sistemine hazırlık çalışması yapılmıştır. Gerçek zamanlı EMG verileri QNET02697-MYOELECTRIC eğitim seti ile alınmış ve LabVIEW programı ile ara yüzü tasarlanmıştır. Böylece, rehabilitasyon sistemleri için araștırma ve eğitim altyapısına katkı oluşturulabilecektir.

\section{Motor Control Setup Design with Determining Threshold Level from EMG Data Using Arduino and LabVIEW}

\section{Keywords}

EMG,

LabVIEW,

Arduino,

Rehabilitation,

\begin{abstract}
Electromyography (EMG) signal analysis in biomedical signals is a popular field of research and it is very important for rehabilitation design systems. The methods used in this study are as follows; i) nerve stimulation of the skeletal muscle over the human to obtain EMG data of real-time hand fingers, ii) determination of hand opening and closing movements threshold levels from obtained EMG signals by histogram method and iii) real time DC motor controls via Arduino using threshold voltage level. This study provided the preparation of the rehabilitation system with an Arduino-based circuit using specified threshold voltages. Real-time EMG data was acquired with the QNET02697-MYOELECTRIC training kit and the graphical user interface of the system was designed with the LabVIEW program. Thus, contribution to the research and education infrastructure for the rehabilitation systems might be established.
\end{abstract}

\section{Giriş}

Elektromiyografi (EMG) sinirler ve kaslardan alınan elektriksel potansiyellerin ölçüldüğü bir biyomedikal işarettir. Kasların kasılma ve dinlenmede oluşturduğu elektrik sinyallerinin değerlendirilmesiyle elde edilir. Sinirleri ve kasları etkileyen hastalıkların tanısında kullanılmaktadır [13]. Biyomedikal cihaz tasarımında kullanılmakla birlikte fizik tedavi ve rehabilitasyon çalışmalarında EMG verileri gözlenerek hastaların iyileşme durumu kontrol edilebilmektedir. EMG işaretlerinden elde edilen anlaml parametreler ve bilgilerle robot kontrolü de yapılabilmektedir. Robotik uzuv tasarımında EMG verilerinden yararlanılmaktadır [4, 5].

EMG işaretinde kas hareketleri farklı membran potansiyellerinden oluşmaktadır. $\mathrm{Bu}$ potansiyellere bakılarak kasın durumu incelenir ve EMG işaretleri çeşitli yöntemler sınıflandırılarak anlamlı sonuçlar ve parametreler elde edilebilir [6]. Canlı vücudunu gözlemlemek ve rehabilitasyon anlamında kontrol etmek yaygın olan uygulamalı araştırma alanıdır. $\mathrm{Bu}$ uygulamalarda EMG işaretleri, sıcaklık ve nem gibi verileri Arduino tabanlı donanımlar ve LabVIEW gibi 
yazılımlar ile grafik ara yüzler aracılığıyla izlemek ve anlamlı parametreler bulmak mümkündür [7-10].

EMG sinyalleri H-H (Hodgkin-Huxley) matematiksel modeli kullanılarak yapay olarak da üretilmektedir. Yapay olarak üretilen bu EMG sinyalleri kullanılarak DC motor kontrolü yapılmıştır [11]. Bu çalışmada gerçek zamanlı veriler farklı insanlardan ölçülen EMG verilerinden el parmaklarının açık ve kapalı eşik gerilimleri bulunmuştur. Bu sonuçlardan gerçek bir DC motorun kontrolü yapılmıştır.

Çalışmanın geri kalan bölümleri sırasıyla dört bölüm olarak sunulmuştur. İkinci bölümde iskelet kasının sinir yolu ile uyarılması, kasın kasılma sırasındaki biyolojik özellikler açıklanmıştır. Ayrıca EMG sinyalinde eşik değerinin belirlenmesi ve gerçek zamanlı EMG verilerinin kasılma ve dinlenme eşik gerilimlerinin elde edilmesi ve kontrol kartı hakkında bilgilendirme yapılmıştır. Üçüncü bölümde gerçek zamanlı veri ölçümü ve DC motor uygulamasında elde edilen bulgular anlatılmıștır. Dördüncü bölümde tasarlanan sistem üzerinde elde edilen sonuçlar değerlendirilmiştir.

\section{Materyal ve Metot}

\section{1. İskelet kasının sinir yolu ile uyarılması}

İskelet kası, sinir sisteminden gelen, sinir lifleri motor nöron ile uyarılır. Motor nöron ucundan salgılanan hormon sinir-kas bağlantısı arasına yayılır. Bu hormon aynı zamanda kas zarının (sarkolemma) uyarılmasını sağlar. Uyarılmış kasın sarkoplazmik retikulumundan $\mathrm{Ca}++$ iyonları ile magnezyum iyonları yer değiștirir. Ca++ iyonları aktin ve miyozin arasına yayılır ve kasın kasılması gerçekleşir. Kas dinlenme durumundayken $\mathrm{Ca}++$ iyonları sarkoplazmik retikulum içerisinde yer alır. Kasın kasılma anındaki membran potansiyeli Şekil 1'de gösterilmiştir.

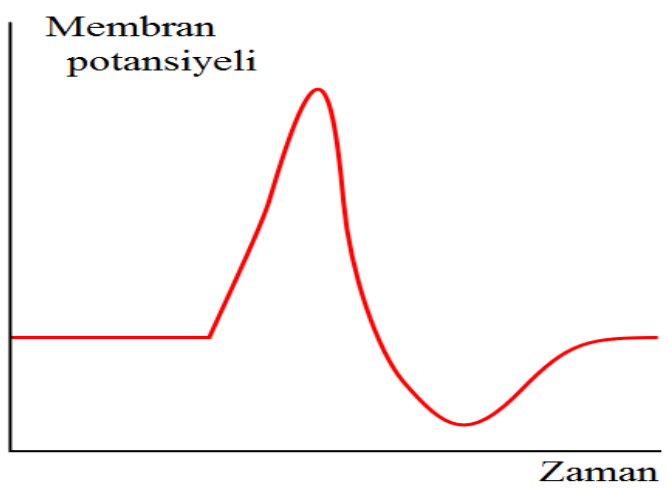

Şekil 1. Membran potansiyeli [12]

Kas gevşemeden uyarılar aşırı hızda gelmeye devam ederse, kas gevşemeden sürekli kasılır. Bu olaya tetanizasyon denir [1-3]. Her bir kas içindeki aksiyon potansiyellerini EMG ile kaydedebiliriz. Kaydedilen potansiyellerin süresi ve genliğindeki farklılıklar EMG verisinin nöroloji ve algolojide tanı amaçlı kullanılabilir [1].

Bu çalışmada, gerçek zamanlı EMG verileri Şekil 2'de gösterilen QNET02697 MYOELECTRIC eğitim setinden alınmıștır [13]. Bu sette, LabVIEW programı kullanıcı ve blok diyagramı olmak üzere iki ayrı ara yüzden oluşur. Kullanıcı ara yüzü olarak adlandırılan ön panelde sistemi kullanacak kişiyi yönlendiren cihazlar yer alır. Blok diyagramı ise grafiksel programlamanın yapıldığı kısımdır. LabVIEW yazılımında kullanıcı ön paneli kullanırken blok diyagram arka planda tüm işi yapar ve alınan verileri kaydeder, hafızaya alır. LabVIEW yazılımda program yazarken, yazılımcı hem ön paneli hem de blok diyagramının tasarımını yapar. LabVIEW yazılımı komut satırları yerine sanal cihazları, hazır blokları kullanır. $\mathrm{Bu}$ da yazılımciya program yazma, hata ayıklama kolaylığı sağlar.

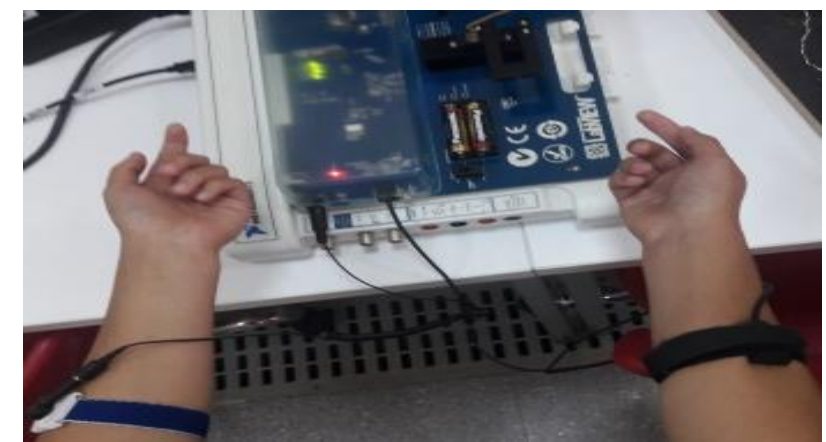

Şekil 2. Gerçek zamanlı EMG verilerinin alınması [13]

\subsection{EMG sinyalinde eşik değerin belirlenmesi}

DC motor hareket etmesi için belirli bir eșik değeri gerekmektedir. Bu eşik değeri ise alınan EMG verisine göre belirlenmektedir. Elin açma ve kapama hareketlerine göre eşik değeri belirlenmiştir. Şekil 3'de elin açma ve kapama hareketleri gösterilmektedir. (a)

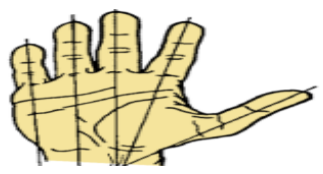

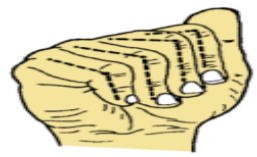

(b)
Şekil 3. a) Elin açma hareketi b) Elin kapama hareketi [14]

Eşik değeri 3 kadın 2 erkek olmak üzere 5 kişiden alınan EMG verilerine göre belirlenmiştir. Kişilerden önce elin açma durumundayken daha sonra da elin kapama durumundayken bir dakika süreyle EMG verileri alınmıştır. Alınan bu EMG kayıtlarının elin açma ve kapama hareketleri için ayrı ayrı histogramları hesaplanmıştır. Her iki el hareketi için ayrı ayrı çıkarılan histogramların maksimum ve minimum değerleri hesaplanmıştır. Elde edilen minimum ve maksimum değerlerinin ortalaması alınarak eșik değeri belirlenmiștir [6] . Şekil 4'de bir denekten alınan EMG verileri ve alınan EMG verilerinin histogramları gösterilmiştir. Elin açma 
hareketinin histogramının minimum ve maksimum değerleri $-0,0850 \mathrm{~V}$ ve $0,0815 \mathrm{~V}$ 'dur. Elin kapama hareketinin histogramının minimum ve maksimum değerleri -1,0180 V ve 1,0940 V 'dur. Elin kapama hareketinde kasların kasılması gerçekleşir. Yapılan bu çalışmada elin kapama hareketinde DC motor aktif hale gelmektedir. Bu nedenle DC motorun aktif hale gelebilmesi için seçilen eşik değeri $1 V^{\prime}$ dur.
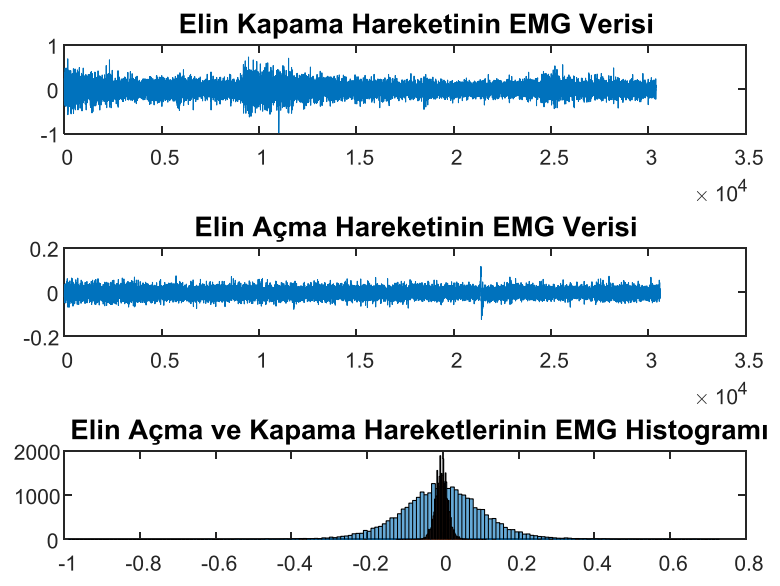

Şekil 4: Bir denekten alınan elin açma ve kapama EMG verileri ve bu verilerinin histogramları

\subsection{EMG verisinin toplanması, işlenmesi ve kontrol kartı}

Bu çalışmada, QNET-02697 MYOELECTRIC eğitim seti ile alınan gerçek zamanlı EMG verileri ile Arduino tabanlı DC motor devresinin kontrolü sağlanmıştır. $\mathrm{Bu}$ uygulama için tasarlanan Arduino tabanlı DC motor devresi Şekil 5'de gösterilmiştir.

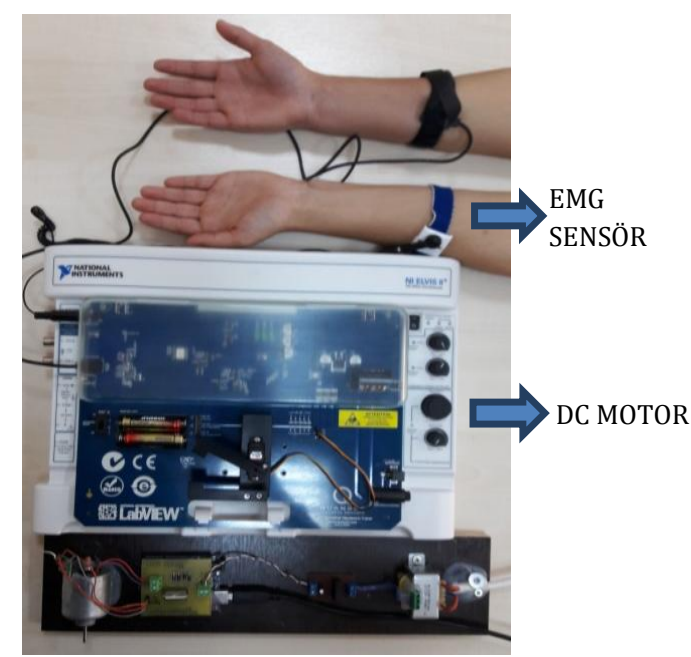

Şekil 5: Sistemin gerçeklenmiş hali

Bu çalıșmada LabVIEW yazılımı ve Arduino tabanlı DC motor kontrol devresinin tasarımı ve uygulamanın gerçeklenmesi Intel ${ }^{\circledR}$ Core $^{\mathrm{TM}}$ i5-3330M CPU @ 3 GHz işlemci 4 GB RAM sahip bilgisayar kullanılmıştır. Gerçek zamanlı EMG veri setinden alınan verilere göre DC motoru kontrol etmek için Arduino (ATmega328P) mikro denetleyicinin ara birim olarak kullanıldığı elektronik devre kullanılmıştır. Bu çalışmada, DC motor milinin bir dakikada içinde tur sayısı tasarlanan mikro denetleyici kart üzerinden kullanıcı ara yüzüne iletilir. LabVIEW yazılımı aracılığı ile grafiksel kodları ile yazılmış uygulama programında ișlendikten sonra kullanıcı ara yüzünde görsel olarak izlenir ve kayıt edilir. Arduino mikro denetleyicinin özellikleri Tablo 1 'de verilmiştir.

Tablo 1. Arduino özellikleri[15]

\begin{tabular}{cc}
\hline Özellikler & Sayısal değerleri \\
\hline Dijital I/O Pinleri & 14 \\
Analog Giriş Pinleri & 6 \\
Çalışma Voltajı & $5 \mathrm{~V}$ \\
SRAM & $2 \mathrm{~KB}$ \\
EEPROM & $1 \mathrm{~KB}$ \\
Çalışma frekansı & $16 \mathrm{MHz}$ \\
\hline
\end{tabular}

\section{Bulgular}

Setten alınan gerçek zamanlı EMG verileri Şekil 6'da gösterilmiştir. Alınan bu gerçek zamanlı EMG verileri insan kolu üzerine bağlı olan algılayıcılar aracılığıyla kasın kasılması üzerine ölçülmüş ve belirlenen eşik seviyesine göre DC motorun kontrol edilmesini sağlamıștır. Başka bir anlatımla, kasın kasılma anındaki maksimum voltaj değerinde DC motor aktif hale gelmektedir.

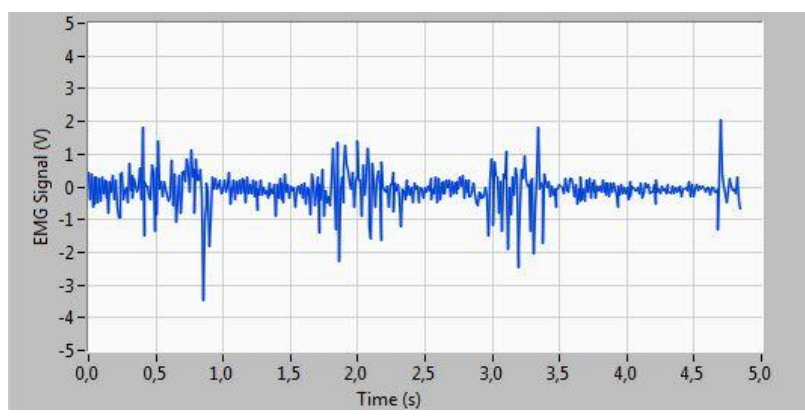

Şekil 6: Alınan gerçek zamanlı bir EMG verisi

EMG verisinin alınıp DC motor giriș sinyali olarak işlenmesi "VISA" kütüphanesi ile sağlanmıștır. Alınan EMG sinyali yükseltilip DC motora giriş olarak uygulanmaktadır. Alınan EMG sinyaline DC motor tepkisi bir saniye gecikmeli olarak verilmektedir. Ayrıca 1V eșik gerilimi olarak seçilmiștir. DC motor $1 \mathrm{~V}$ üzerinde gelen EMG sinyallerinde harekete geçmektedir. 1V üzerindeki sinyallerde DC motor aktif hale gelmekte ve motor hızı da gelen EMG sinyalinin gerilime bağlı olarak değișmektedir. Sistemin çalışırken LabVIEW programındaki blok diyagramı Şekil 7'de kullanıcı ara yüzü ise Şekil 8'de gösterilmiştir.

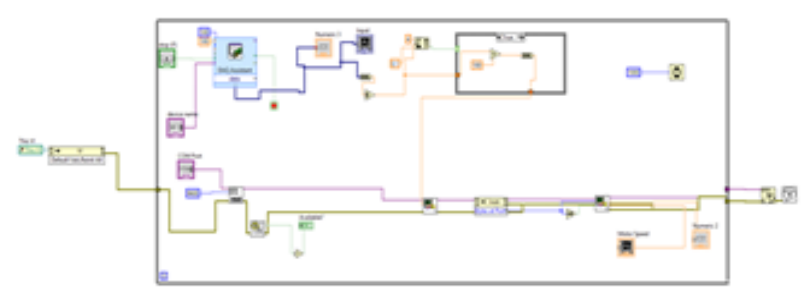

Şekil 7. Sistemin LabVIEW blok diyagramı 


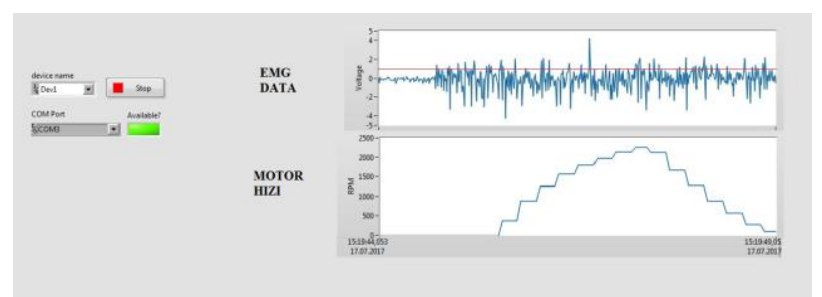

Şekil 8: Sistemin LabVIEW kullanıcı ara yüzü

\section{Sonuçlar}

Gerekli prosedürler gerçekleştirilip tüm bağlantılar yapıldıktan sonra QNET-02697 MYOELECTRIC eğitim seti ile insan kolu üzerinden EMG verileri elde edilmiştir. Elde edilen EMG veri setlerine göre kasın kasılması sırasında oluşan maksimum gerilim değeriyle belirlenen eşik seviyesinde DC motor aktif hale gelmektedir. Elde edilen veriler neticesinde yaptığımız bu çalıșma robotik uzuv tasarımı için hazırlık çalışması oluşturmaktadır. Basit rehabilitasyon robot tasarımında DC motor kontrolü faydalı olabilecektir. Arduino ișlemcisinin Ethernet, WIFI veya Bluetooth modülleri kullanılarak sistemin uzaktan kontrolü de gerçekleştirilebilir.

\section{Teşekkür}

$\mathrm{Bu}$ çalışma, İzmir Kâtip Çelebi Üniversitesi Bilimsel Araştırma Projesi (proje numarası 2015-ÖDL-MÜMF0004) tarafından kısmen desteklenmiş ve çalışmanın ilk sonuçları XX. Biyomedikal Mühendisliği Ulusal Toplantı (Uluslararası Katılımlı) BIYOMUT 2016'da "Gerçek Zamanlı EMG Verileri ile DC Motor Kontrolü" bildirisi ile sunulmuştur.

\section{Kaynakça}

[1] Guyton, A.C., Hall, J.E. 2006. Medical Physiology El Kitabı, 11. Basım, W.B. Saunders Company.

[2] Khushaba, R.N., Al-Timemy, A., Kodagoda, S., Nazarpou, K. 2016. Combined influence of forearm orientation and muscular contraction on EMG pattern recognition. Expert Systems with Applications, 61, 154-161.

[3] Bhardwaj, N., Chopra, P., Rania A., Agarwal, S. 2016. Extraction of EMG signals in a software compatible format from an online database using WFDB package. Perspectives in Science, 8, 767-769.

[4] Mayetin, U., Küçük, S., Şayli, Ö. 2015. EMG Kontrollü Mobil Robot Uygulaması. Tıp Teknolojileri Ulusal Kongresi, 15-18 Ekim, Muğla, 145-148.

[5] Şenli K. 2011. EMG (Elektromiyografi) Kontrollü Protez Kol Tasarımı. Dokuz Eylül Üniversitesi, Fen Bilimleri Enstitüsü, Yüksek Lisans Tezi, 54s, İzmir.

[6] Akben, S. B. 2015. Kavrama ile İlişki El Hareketlerinin EMG İşaretleri Kullanılarak
Sinıflandırılması. 19. Ulusal Biyomedikal Mühendisliği Toplantısı, DOI: 10.1109/BIYOMUT.2015.7369445.

[7] Joshi, N. P., Patel, P. V. 2014. LabVIEW and WebServer based Human Body Monitoring System. International Journal of Advanced Research in Electrical, Electronics and Instrumentation Engineering 3(5), 9356-9362.

[8] O’Brien, E. M., Rogge, R. D. 2002. LabVIEW Usage As Part of The Biomedical Engineering Senior Design Experience. In Engineering in Medicine and Biology, 24th Annual Conference and the Annual Fall Meeting of the Biomedical Engineering Society EMBS/BMES Conference, Proceedings of the Second Joint, 3, 2599-.

[9] Călinoiu, D., Ionel, R., Lascu, M., Cioablă, A. 2014. Arduino and LabVIEW in Educational Remote Monitoring Applications. IEEE Frontiers in Education Conference Proceedings, 1-5.

[10] Zulkifli, N. S. A., Che Harun, F. K., Azahar, N. S. 2012. XBee Wireless Sensor Networks for Heart Rate Monitoring in Sport Training. In Biomedical Engineering (ICoBE), International Conference, 441-444.

[11] Şahin, S., Bayraktar, M., Evren Şahin, K. 2016. Design and Implementation of Experimental Setup For Neural Data Based Stimulator By Using LabVIEW. International Multilingual Academic Journal, 2(1).

[12] Şahin, S., Bayraktar, M., Kavur, A. E., Evren Şahin, K. 2016. Gerçek Zamanlı EMG Verileri ile DC Motor Kontrolü. XX. Biyomedikal Mühendisliği Ulusal Toplantı (Uluslararası Katılımlı) BIYOMUT 2016, Seferihisar İzmir, 136-138.

[13] Elin Açma ve Kapama Hareketleri. 2016. http://docplayer.biz.tr/5407716-Ortopedgkprotez-ortez.html (Erişim Tarihi: 10.12.2016).

[14] Arduino Uno Özellikleri. 2016. https://www.arduino.cc/en/Main/ArduinoBoar dUno (Erişim Tarihi: 10.07.2016). 